\title{
Ambiguity Solution of Relative Position of Carrier Phase Differential
}

\author{
Xuke Yang, Hao Jiang \& Dong Liu \\ School of Rocket Force University of Engineering, Xi'An 710025, China
}

ABSTRACT: In view of the ambiguity solution is the technical difficulty in the relative position of the carrier phase differential. This paper introduces the principle of the carrier phase differential relative positioning and the process of integer ambiguity solution in detail. The ambiguity is solved by LAMBDA (the least-square ambiguity decorrelation adjustment) method in a short baseline, and the float solution and the fixed solution of the ambiguity and the baseline are compared. The result show that the carrier phase differential positioning can reach the positioning accuracy of centimeter level.

KEYWORD: relative positioning; carrier phase; integer ambiguity; float solution

\section{INTRODUCTION}

In recent years, with the progress of science and technology and expansionary demand of application, the upsurge of GNSS (global navigation satellite system) application and development is spring up in domestic market. Not only the surveying and mapping industry, other related industries such as transportation, aerospace, meteorology, exploration, mining and other industries is also being carried out satellite navigation application and development (LIU Ji-yu, 2003). Due to the characteristics and the application of GNSS signal itself, prompting the differential relative positioning technologies available, according to the types of measurement can be classified as the position difference, pseudo-range differential and carrier phase differential. Generally, the dynamic real-time precise relative positioning use carrier phase differential for it is high precision of centimeter level.

\section{THE PRINCIPLE OF CARRIER PHASE DIFFERENTIAL POSITIONING}

The model of carrier phase differential positioning composed of two receivers. One of them as a base station, the other one as a mobile station. The original observation data is transformed to the mobile station by the base station instantly, and construct the double difference carrier phase observation equation. The double difference carrier phase can weaken or even eliminate the influence of orbit error, clock error, atmospheric error and other errors, real-time data processing, thereby, determine the location and speed of the mobile station in real time (XIE Gang, 1992) (Capkun S et al, 2002).

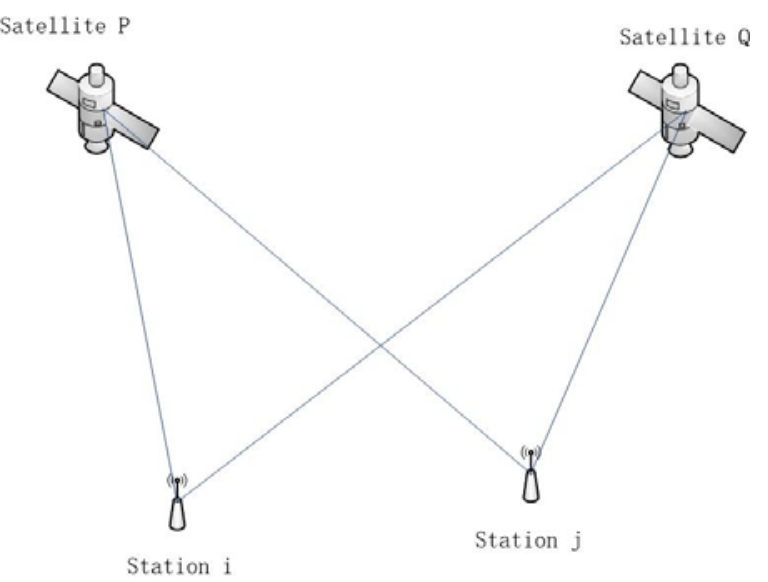

Fig. 1 Double difference relative positioning schematic

In the carrier phase differential positioning, carrier phase observation equation of the carrier phase of the receiver $j$ observation satellite $P$ shown in Figure 1.

$$
\varphi_{j}^{P} \lambda=\sqrt{\left(X^{P}-X_{j}\right)^{2}+\left(Y^{P}-Y_{j}\right)^{2}+\left(Z^{P}-Z_{j}\right)^{2}}-C V_{t_{j}}+C V_{t_{P}}-N_{j}^{P} \lambda-\left(V_{\text {ion }}\right)_{j}^{P}-\left(V_{\text {trop }}\right)_{j}^{P} \text {. }
$$


Where

$\varphi_{j}^{P}$ is the carrier phase observations of the receiver $j$ observation satellite $P .\left(X^{P}, Y^{P}, Z^{P}\right)$ is coordinate of satellite $P .\left(X_{j}, Y_{j}, Z_{j}\right)$ is coordinate of receiver $j$.c is electromagnetic wave propagation velocity. $V_{t_{j}}$ is the receiver clock error of receiver $j \cdot V_{t_{P}}$ is the receiver clock error of satellite $P . N_{j}^{P}$ is the carrier phase ambiguity of the receiver $j$ observation satellite $P .\left(V_{\text {ion }}\right)_{j}^{P}$ is the ionospheric errors between receiver $j$ and satellite $P .\left(V_{\text {trop }}\right)_{j}^{P}$ is the tropospheric errors between receiver $j$ and satellite $P$.

The geometric distance of the receiver $j$ to the satellite $P$ is assumed to

$\rho_{j}^{P}=\sqrt{\left(X^{P}-X_{j}\right)^{2}+\left(Y^{P}-Y_{j}\right)^{2}+\left(Z^{P}-Z_{j}\right)^{2}}$.
When the initial $j$ of the receiver is $\left(X_{j 0}, Y_{j 0}, Z_{j 0}\right)$, the correction for the coordinate is $\left(\delta X_{j}, \delta Y_{j}, \delta Z_{j}\right)$. The distance of initial coordinate to the satellite $P$ is

$$
\rho_{j 0}^{P}=\sqrt{\left(X^{P}-X_{j 0}\right)^{2}+\left(Y^{P}-Y_{j 0}\right)^{2}+\left(Z^{P}-Z_{j 0}\right)^{2}} .
$$

Thus direction cosine vector of the receiver $j$ to the satellite $P$ is

$$
\begin{aligned}
& \frac{\partial \rho_{j}^{P}}{\partial X^{P}}=\frac{X^{P}-X_{j 0}}{\rho_{j 0}^{P}}=l_{j}^{P}, \frac{\partial \rho_{j}^{P}}{\partial Y^{P}}=\frac{Y^{P}-Y_{j 0}}{\rho_{j 0}^{P}}=m_{j}^{P}, \\
& \frac{\partial \rho_{j}^{P}}{\partial Z^{P}}=\frac{Z^{P}-Z_{j 0}}{\rho_{j 0}^{P}}=n_{j}^{P}
\end{aligned}
$$

The linearization of the carrier phase observation equation in $\left(X_{j 0}, Y_{j 0}, Z_{j 0}\right)$ is

$$
\varphi_{j}^{P} \lambda=\rho_{j 0}^{P}-\left(l_{j}^{P}, m_{j}^{P}, n_{j}^{P}\right)\left(\delta X_{j}, \delta Y_{j}, \delta Z_{j}\right)-C V_{t_{j}}+C V_{t_{P}}-N_{j}^{P} \lambda-\left(V_{\text {ion }}\right)_{j}^{P}-\left(V_{\text {trop }}\right)_{j}^{P}
$$

In the relative position of the carrier phase, the reference station's coordinate is assumed to know $\left(X_{i 0}, Y_{i 0}, Z_{i 0}\right)$. The coordinates of the mobile station are $\left(X_{i}, Y_{i}, Z_{i}\right)$. And take the initial coordinates of the mobile station as the reference station coordi- nates $\left(X_{i 0}, Y_{i 0}, Z_{i 0}\right)$. The mobile station coordinates correction vector is $\left(\delta X_{j}, \delta Y_{j}, \delta Z_{j}\right)$. Thus, in the case of the same carrier frequency, the observation equations of the reference station and the mobile station are respectively expressed as

$$
\varphi_{j}^{P} \lambda=\rho_{i 0}^{P}-c V_{t_{i}}+c V_{t_{P}}-N_{i}^{P} \lambda-\left(V_{\text {ion }}\right)_{i}^{P}-\left(V_{\text {trop }}\right)_{i}^{P} \text {. }
$$

$$
\varphi_{j}^{P} \lambda=\rho_{i 0}^{P}-\left(l_{i}^{P}, m_{i}^{P}, n_{i}^{P}\right)\left(\delta X_{j}, \delta Y_{j}, \delta Z_{j}\right)-c V_{t_{j}}+c V_{t_{P}}-N_{j}^{P} \lambda-\left(V_{\text {ion }}\right)_{j}^{P}-\left(V_{\text {trop }}\right)_{j}^{P} .
$$

In the case of short baseline, two stations $i, j$ is approximately equal to the ionosphere and troposphere correction parameters.the single difference observation equation can be obtained

$\Delta \varphi_{i j}^{P} \lambda=\rho_{i 0}^{P}-\left(l_{i}^{P}, m_{i}^{P}, n_{i}^{P}\right)\left(\delta X_{j}, \delta Y_{j}, \delta Z_{j}\right)-c V_{t_{i}}+c V_{t_{P}}-\Delta N_{i j}^{P} \lambda$

Where

$\Delta \varphi_{i j}^{P}=\varphi_{i}^{P}-\varphi_{j}^{P}, \Delta N_{i j}^{P}-N_{i}^{P}-N_{j}^{P}$.

The differential equations of the two receivers with respect to the single difference equations of the two satellites can be obtained, which can be compared with the two satellites.

$\Delta \varphi_{i j}^{P Q} \lambda=\left(l_{i}^{P}-l_{i}^{Q}, m_{i}^{P}-m_{i}^{Q}, n_{i}^{P}-n_{i}^{Q}\right)\left(\delta X_{j}, \delta Y_{j}, \delta Z_{j}\right)-\Delta N_{i j}^{P Q} \lambda$.

Where

$\Delta \varphi_{i j}^{P Q}=\Delta \varphi_{i j}^{P}-\Delta \varphi_{i j}^{Q}, \quad \Delta N_{i j}^{P Q}=\Delta N_{i j}^{P}-\Delta N_{i j}^{Q}$.

\section{INTEGER AMBIGUITY RESOLUTION}

In this paper, the LAMBDA method is proposed in document (TEUNISSEN PJG, 1995) to construct the search space by using floating point ambiguity and covariance matrix in order to determine the integer ambiguity (ZHAO Bei et al, 2008) (LU Xian-jian, 2008). But not guaranteed to get the correct solution, in order to obtain the accurate ambiguity, of double difference ambiguity float solution of integer transform related drop, to reduce the fuzzy correlation degree between components. Then the transform order used under the condition of fuzzy floating point degree and its covariance matrix structure search space, based on ambiguity of residual sum of squares and minimum criteria determine the integer ambiguity, the method has attracted much attention due to their (TEUNISSEN PJG et al, 1997) (WANG 
Zi-ru et al, 2000) owing to its high search efficiency. The solution process is shown in figure 2 .

(1)using the least square method to solve ambiguity float solution.

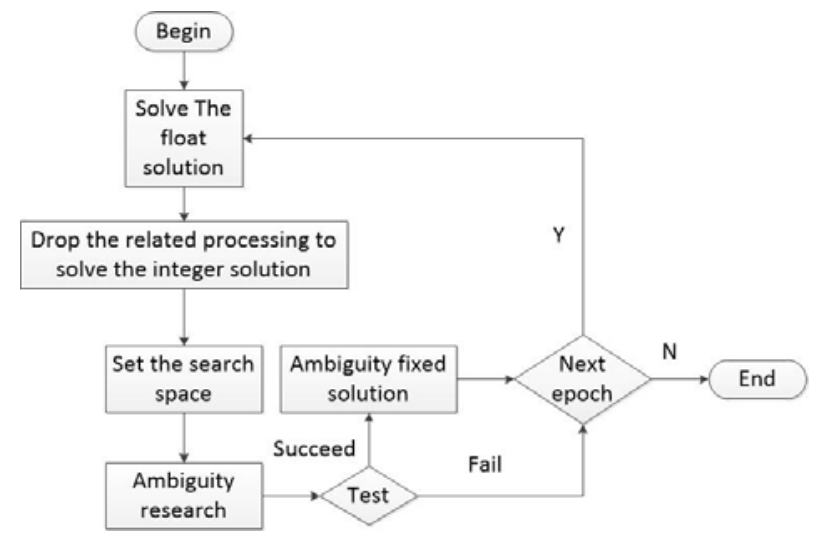

Fig. 2 block diagram of ambiguity

Rewrite the formula (8) as the following

$y=A a+B b=\left[\begin{array}{ll}A & B\end{array}\right]\left[\begin{array}{l}a \\ b\end{array}\right]$.

Take $G=\left[\begin{array}{ll}A & B\end{array}\right]$, Float solution and the covariance matrix of least-squares were

$\left[\begin{array}{l}\hat{a} \\ \hat{b}\end{array}\right]=\left(G^{T} G\right)^{-1} G^{T} y$

$Q_{[\hat{a}, \hat{b}]}=\left(G^{T} G\right)^{-1}=\operatorname{cov}\left[\begin{array}{c}\hat{a} \\ \hat{b}\end{array}\right]=\left[\begin{array}{cc}Q_{\hat{a}} & Q_{\hat{a}, \hat{b}} \\ Q_{\hat{b}, \hat{a}} & Q_{\hat{b}}\end{array}\right]$

Where

$b=\left(\delta X_{j}, \delta Y_{j}, \delta Z_{j}\right), \quad a=\left(\Delta N_{i j}^{L_{1} Q}, \cdots, \Delta N_{i j}^{L_{k} Q}\right)$

satellite is assumed as a reference satellite),

$A=\lambda_{i} I_{K}, \quad B=\left(l_{i}^{P}-l_{i}^{Q}, m_{i}^{P}-m_{i}^{Q}, n_{i}^{P}-n_{i}^{Q}\right)_{K \times 3}$

$Q_{\hat{a}}$ representative of the covariance matrix of

$\hat{a} \cdot Q_{\hat{b}}$ representative of the covariance matrix of

$\hat{b} \cdot Q_{\hat{a}, \hat{b}}$ representative of the cross-covariance matrices of $\hat{a}, \hat{b}$.

(2)The second step is determined on the basis of the float solution, the integer transform, ambiguity program under the conditions used to construct the objective function.

$\min _{a}(a-\hat{a})^{T} Q_{\hat{a}}^{-1}(a-\hat{a})$.

And make value of the objective function to be minimized and calculating ambiguity integer solution.

(3)After the integer ambiguity resolution is then calculated according to the following formula.

$\bar{b}=\hat{b}-Q_{\hat{b} \hat{a}} Q_{\hat{a}}^{-1}(\hat{a}-\bar{a})$
Solving coordinate baseline vector, complete differential positioning. Where, $\bar{b}$ representative the estimate of location parameters fixed solution. Its corresponding covariance matrix is $Q_{\bar{b}} \cdot Q_{\hat{b} \hat{a}}$ is covariance. $\bar{a}$ is fixed solution of Ambiguity.

\section{TEST ANALYSIS}

This paper uses a static set of short baseline data, the baseline length is 5.46 meters, the epoch number for 35 epochs. In the relative position of the short baseline carrier phase, the receiver clock error, satellite clock error, ionosphere error and troposphere error are almost completely eliminated, which can get a high positioning accuracy.

Figure 3 shows the relationship between the integer solution and the integer solution of the integer ambiguity of the number 13 satellite of GPS. At the beginning of the solution, the float solution with integer solutions of the difference is relatively large, with the increase of the number of epochs, integer solution is more stable, about 25 epochs in the ambiguity of fixation. The relationship between the ambiguity and integer solutions of other satellites is similar to that in Figure 3.

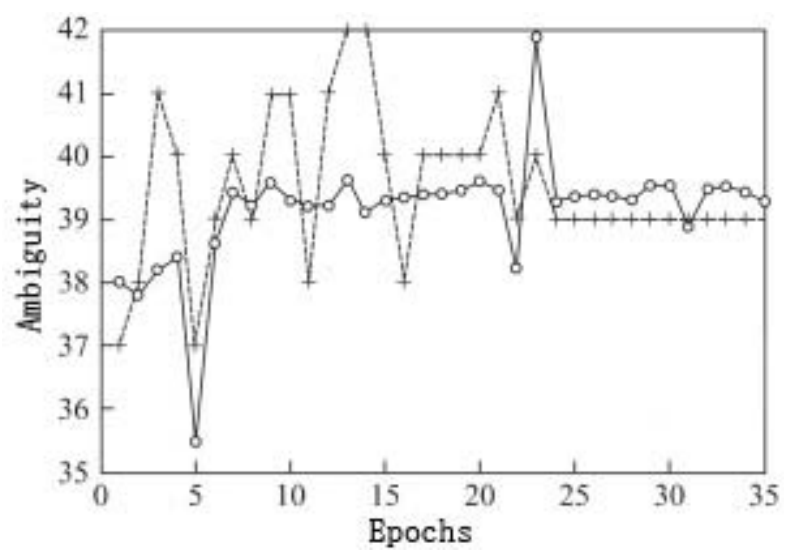

Fig.3Comparison of ambiguity float solution with integer solution of the number 13 satellite of GPS

Figure 4 show the baseline vector of the floating point solution and the fixed solution. From Figure 4, show that the positioning at the beginning, float solution and the relatively large difference in the fixed solution, after 10 epochs, float solution fixed to the 5.45 centimeters, close to the stationary solution. Comparison of measured values and positioning results found that the positioning accuracy is up to centimeter level. 


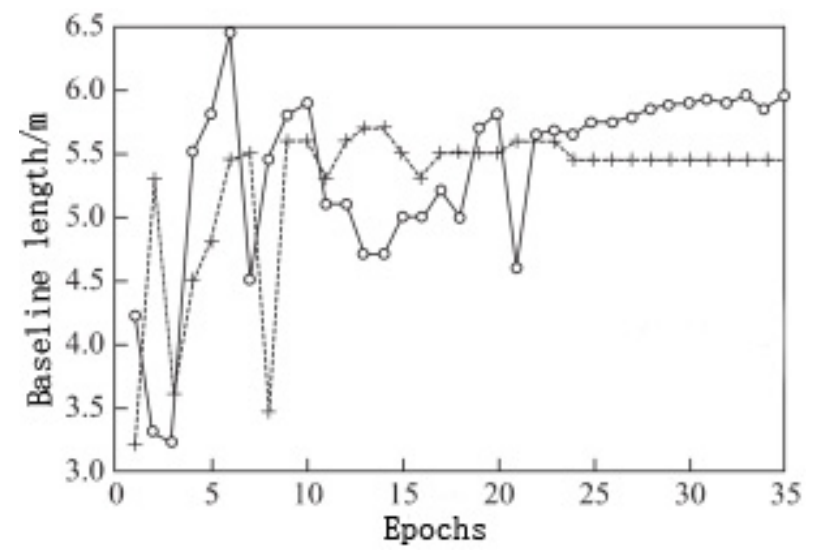

Fig. 4 the baseline vector of the floating point solution and the fixed solution

\section{CONCLUSION}

The difficulty of the relative position of the carrier phase difference is the solution of integer ambiguity. In this paper, the LAMBDA method is used to solve the integer ambiguity of the static short baseline. The lambda method reduces the correlations between variables and accelerate the search speed, only need a few observation epochs will be able to solve calculated ambiguity. This method is very suitable for precision positioning of static and dynamic positioning.

\section{REFERENCES}

Capkun S, Hamdi M, Hubaux J-P. GPS-Free positioning in mobile ad-hoc networks. Cluster Computing, 2002, pp. 157167.

LIU Ji-yu. The positioning principle and method of GPS satellite navigation[M],Science Press, Beijing,2003.

LU Xian-jian. Study of GPS integer ambiguity resolution analysis theory and method[D],Guilin Institute of Technology, Guilin, 2008.

TEUNISSEN PJG.DE JONGE P J, TIBERIUS C C J M. The Least-squares Ambiguity Decorrelation Adjustment: Its Performance on Short GPS Baselines and Short Observation Spans[J]. Journal of Geodesy, 1997, pp. 589-602.

TEUNISSEN PJG. The Invertible GPS Ambiguity Transformation [J].Manuscripta Geodaetica, 1995, pp. 489-497.

WANG Zi-ru, LI Feng-bin. Review for the problem of GPS Ambiguity solution[J], Northeast mapping, 2000, pp. 14-16.

XIE Gang. GPS Principle and receiver design. Surveying and Mapping Press, Beijing, 1992.

ZHAO Bei, WANG Fei-xue, SUN Guang-fu, et al. Integer Z transform algorithm of LAMBDA ambiguity solver methods [J], Journal of MISSILES AND GUIDANCE, 2008, pp. 254-257. 